\title{
Variational characterization for eigenvalues of Dirac operators.
}

\author{
Jean Dolbeault \& Maria J. Esteban \\ Ceremade (URA CNRS 749) \\ CNRS et Université Paris-Dauphine \\ Place Maréchal Lattre de Tassigny \\ F-75775 Paris Cedex 16
}

\author{
Eric Séré \\ Département de Mathématiques \\ Université de Cergy-Pontoise \\ 2, Av. Adolphe Chauvin \\ F-95302 Cergy-Pontoise Cedex
}

\begin{abstract}
In this paper we give two different variational characterizations for the eigenvalues of $H+V$ where $H$ denotes the free Dirac operator and $V$ is a scalar potential. The first one is a min-max involving a Rayleigh quotient. The second one consists in minimizing an appropriate nonlinear functional. Both methods can be applied to potentials which have singularities as strong as the Coulomb potential.
\end{abstract}

AMS Subject Classification: 49 R 05, 49 R 10, 49 R 20, 49 S 05, 47 A 75, 81 Q 10, 35 P 05, 35 Q 75, 35 A 15.

Key Words : Dirac operators, relativistic quantum mechanics, eigenvalues, min-max, minimization, Rayleigh-Ritz technique.

\section{1 - Introduction.}

The Dirac operator is a first order operator which has the form

$$
H=-i c \hbar \vec{\alpha} \cdot \vec{\nabla}+m c^{2} \beta
$$

where $c$ denotes the speed of light, $m>0$ the mass of the electron, $\hbar$ is Planck's constant, $\vec{\alpha} \cdot \vec{\nabla}=\sum_{k=1}^{3} \alpha_{k} \partial_{k}$ and $\alpha_{k}$ are the Pauli-Dirac matrices,

$$
\beta=\left(\begin{array}{cc}
I & 0 \\
0 & -I
\end{array}\right), \quad \alpha_{k}=\left(\begin{array}{cc}
0 & \sigma_{k} \\
\sigma_{k} & 0
\end{array}\right) \quad(k=1,2,3) .
$$


The $2 \times 2$ matrices $\sigma_{k}$ are the Pauli matrices:

$$
\sigma_{1}=\left(\begin{array}{ll}
0 & 1 \\
1 & 0
\end{array}\right) \quad, \quad \sigma_{2}=\left(\begin{array}{cc}
0 & -i \\
i & 0
\end{array}\right) \quad, \quad \sigma_{3}=\left(\begin{array}{cc}
1 & 0 \\
0 & -1
\end{array}\right)
$$

All throughout this paper, we shall work with a system of physical units such that $m=c=\hbar=1$.

In this paper we are interested in perturbed Dirac operators of the form $H+V, V$ being a scalar potential. Our goal is to find variational characterizations for the eigenvalues of such operators. We hope that our results can be of some interest in a priori estimates, as well as practical computations of atomic energy levels. One expects the eigenvalues of $H+V$ to be critical points (in an appropriate sense) of the Rayleigh quotient

$$
R_{V}(\varphi):=\frac{((H+V) \varphi, \varphi)}{(\varphi, \varphi)},
$$

where $(\cdot, \cdot)$ denotes the inner product in $L^{2}\left(\mathbb{R}^{3}\right)$. If $H+V$ were bounded below, like Schrödinger operators $-\Delta-Z /|x|$ for instance, minimizing the above quotient would give the least eigenvalue (ground state energy). This standard approach fails for Dirac operators: for physically relevant potentials $V$, the Rayleigh quotient is neither bounded from above nor from below (for instance, see [20]). This is the main difficulty we have to face.

Many efforts have been devoted to the characterization and computation of Dirac eigenvalues. Some works deal with approximate potentials acting on 2-spinors. The idea is to write Dirac's equation for the upper spinor in $\psi$ by performing some adequate transformation. Then, different procedures have been produced to find approximate Hamiltonians, which "become exact" in the nonrelativistic limit. For references in this direction see, for instance, Durand [13], Durand-Malrieu [14], van Lenthe-Van Leeuwen-Baerends-Snijders [24].

Other approximate Hamiltonians are constructed by using projectors. One of the first attempts in this direction was made by Brown and Ravenhall. In [7], they proposed the following (usually called the Brown-Ravenhall "nopair") Hamiltonian

$$
B=\Lambda^{+}(H+V) \Lambda^{+}
$$

where $\Lambda^{+}=\chi_{(0, \infty)}(H)$ denotes the projection on the positive spectral subspace of the free Dirac operator.

The projected Hamiltonian $B$ has received a lot of attention concerning the question of the "stability of matter" for relativistic multi-particle systems (see Lieb-Siedentop-Solovej [26]). 
If 0 is not in the spectrum of $H+V$, then instead of defining $B$ as above, one can use the exact positive projector corresponding to $H+V$, denoted by $\Lambda_{V}^{+}$. The new operator

$$
\tilde{B}_{V}=\Lambda_{V}^{+}(H+V) \Lambda_{V}^{+}
$$

coincides with $H+V$ in its positive spectral subspace. For attractive potentials $V$ that are not "too strong", minimizing the Rayleigh quotient for $\tilde{B}_{V}$ yields the smallest positive eigenvalue of $H+V$. This eigenvalue may be interpreted as the ground state energy. Unfortunately, the exact projector $\Lambda_{V}^{+}$is not known a priori. This makes its use difficult in numerical computations. On the contrary, the approximate Brown-Ravenhall Hamiltonian $B$ is known explicitly.

Several recent papers have been devoted to the mathematical study of the Brown-Ravenhall Hamiltonian for one-electron atoms with nuclear charge $Z>0$. Its precise form is

$$
B^{Z}:=\Lambda_{+}\left(H-\frac{Z \alpha}{|x|}\right) \Lambda_{+}
$$

where $\alpha$ is the fine structure constant $\left(\alpha^{-1}=137.037 \ldots\right)$. Hardekopf and Sucher [18], on the basis of numerical computations, conjectured that the operator $B^{Z}$ is strictly positive for $Z<Z_{c}=2 \alpha^{-1} /(\pi / 2+2 / \pi)$, that its first eigenvalue vanishes for $Z=Z_{c}$, and that $B_{Z}$ is unbounded from below for $Z>Z_{c}$. Evans, Perry and Siedentop [16] proved that the operator $B^{Z}$ is bounded from below if and only if $Z \leq Z_{c}$, as predicted in [18]. Tix $[32,33]$ proved that the operator $B^{Z}$ is strictly positive for $Z \leq Z_{c}$ (see also Burenkov-Evans [8]). Note that the integer part of $Z_{c}$ is 124 , strictly below the critical value $Z_{*}=137$, for which the first eigenvalue of the DiracCoulomb Hamiltonian $H-Z \alpha /|x|$ vanishes (see for instance [28, 31]).

One the most important properties of the free Dirac operator $H$ is that

$$
H^{2}=-\Delta+1
$$

Another Hamiltonian having the same property is :

$$
\sqrt{-\Delta+1}
$$

This Hamiltonian is positive definite, and so, it does not pose the problems related to the unboundedness of the Dirac operator. But it is nonlocal, which contradicts the Causality Principle in special relativity and does not preserve 
the symmetry in the $t$ and $x$ variables for the evolution problem (covariance of the equation under the action of Lorentz boosts). Nevertheless, the study of the spectrum of

$$
\sqrt{-\Delta+1}+V
$$

and its use as an approximation model for multi-particle systems is interesting. For references in this direction, let us mention [10, 19, 23, 25] and references therein.

In 1994, Hill and Krauthauser proposed a variational characterization of eigenvalues of $H+V$ based on maximization of quotients like

$$
\frac{(\psi,(H+V) \psi)}{\left(\psi,(H+V)^{2} \psi\right)}
$$

This method works when the spectrum of $H+V$ has a gap around 0 . The mathematical study of this approach has still to be done.

The characterization of the stable eigenvalues of $H+V$ by a minimax method is a quite natural idea and it has been attempted both analytically and numerically : in 1986, Talman [30] proposed a minimax procedure to find the first eigenvalue of $H+V$ for negative potentials $V$ (see also [9]). His proposal consisted in first maximizing in the lower component of the spinor $\psi$, and then minimizing over the upper spinor.

In 1995, in a PDE conference in Toronto (see its proceedings [15]), Esteban and Séré presented a minimax technique (without approximation) inspired of critical point theory. This same result was also presented in the ICDEMP in Atlanta in March 1997. A sequence of min-max levels was introduced in [15]:

$$
\lambda_{k}=\inf _{\substack{F \subset Y^{+} \\ F \text { vector space } \\ \text { dim } F=k}} \sup _{\substack{\varphi \in F \oplus Y^{-} \\ \varphi \neq 0}} \frac{((H+V) \varphi, \varphi)}{(\varphi, \varphi)} .
$$

Here, $Y^{ \pm}$are the spaces $\Lambda^{ \pm}\left(H^{1 / 2}\left(\mathbb{R}^{3}\right)\right)$. The following result was announced: for a class of potentials that includes the Coulomb potentials $-a|x|^{-1} \quad(0<a<1 / 2), \lambda_{k}$ is the k'th positive eigenvalue of $H+V$ (counted with multiplicity). These values of $a$ correspond to one-electron atoms of atomic charge $Z \leq 68$.

Recently, Griesemer and Siedentop ([17]) proved a general minimax theorem on the eigenvalues in a spectral gap of an abstract operator. As an application, they prove that under appropriate conditions on $V$, the sequence 
$\lambda_{k}$ defined in (1.8) coincides with the sequence of positive eigenvalues $\mu_{k}$ of $H+V$. The method of proof in [17], as well as the class of potentials $V$ to which their theorem applies, seems to be quite different from ours: in the case of Coulomb potentials, they only prove that $\lambda_{k} \leq \mu_{k}$. Another interesting result in [17], is that Talman's approach, slightly modified, is indeed mathematically correct for a large class of bounded potentials.

In Section 2 of the present paper, we state and prove some technical results on the Dirac operator. Sections 3 and 4 deal with two variational characterizations of the eigenvalues.

Section 3 is devoted to the study of the minimax procedure (1.8). We give a detailed proof of the results announced in Toronto (see [15]) and in Atlanta. In [15], we could not treat "strong" Coulomb potentials $-a|x|^{-1}$, for $a \geq 1 / 2$, because we were using estimates based on Hardy's inequality

$$
\int \frac{|u|^{2}}{|x|^{2}} \leq 4 \int|\nabla u|^{2}
$$

We are now able to treat all $a<2 /(\pi / 2+2 / \pi) \approx 0.9$. This corresponds to an atomic charge $Z$ up to 124 . The proof is almost the same for these strong fields, we simply replace Hardy's inequality by the very precise estimates of Tix [32, 33] and Burenkov-Evans [8].

The minimization method introduced in Section 4, follows a completely different idea. It comes from the reduction of the Hamiltonian to a 2-spinor function space. The reduced energy functional $J^{+}$is nonlinear, but semibounded. For a large family of operators $V$, the minimization of $J^{+}$provides us with the smallest eigenvalue of $H+V$ (and an associated eigenfunction) as long as it is positive. This yields an optimal result for the Coulomb potential $-Z \alpha /|x|$ : our minimization method works for all $Z<137$, the best condition under which $H-Z \alpha /|x|$ possesses a ground state in $L^{2}\left(\mathbb{R}^{3}\right)$. In a sense, $J^{+}$seems to contain information related to the exact projector $\Lambda_{V}^{+}$in an implicit way. Note that the idea to build a semibounded energy functional had already been introduced by Bayliss and Peel [1] in another context.

Aknowledgement. The authors wish to thank H. Siedentop for his interest in this work. His bibliographical remarks have been very useful.

\section{2 - Generalities on the Dirac operator and auxiliary results.}

In the Fourier domain, the Dirac operator is a multiplication operator 
(by a $4 \times 4$ hermitian matrix) :

$$
\hat{H}(\xi)=\xi \cdot \alpha+\beta,
$$

and it satisfies

$$
\hat{H}^{2}=|\xi|^{2}+1 .
$$

For every $\xi \in \mathbb{R}^{3}, \hat{H}(\xi)$ has two eigenvalues, $\pm \sqrt{|\xi|^{2}+1}$ and one has the following properties :

\section{Lemma 2.1.}

(P1) $H$ is a self-adjoint operator on $L^{2}\left(\mathbb{R}^{3}, \mathbb{C}^{4}\right)$, with domain $\mathcal{D}(H)=$ $H^{1}\left(\mathbb{R}^{3}, \mathbb{C}^{4}\right)$. Its spectrum is $(-\infty,-1] \cup[1,+\infty)$. There are two orthogonal projectors on $L^{2}\left(\mathbb{R}^{3}, \mathbb{C}^{4}\right), \Lambda^{+}$and $\Lambda^{-}=1_{L^{2}}-\Lambda^{+}$, both with infinite rank, and such that

$$
\left\{\begin{array}{l}
H \Lambda^{+}=\Lambda^{+} H=\sqrt{1-\Delta} \Lambda^{+}=\Lambda^{+} \sqrt{1-\Delta} \\
H \Lambda^{-}=\Lambda^{-} H=-\sqrt{1-\Delta} \Lambda^{-}=-\Lambda^{-} \sqrt{1-\Delta}
\end{array}\right.
$$

(P2) The Coulomb potential $W(x)=\frac{1}{|x|}$ satisfies the following Hardytype inequalities :

$$
\begin{aligned}
(\varphi, W(x) \varphi)_{L^{2}} & \leq \frac{\pi}{2}(\varphi, \sqrt{-\Delta} \varphi)_{L^{2}} \leq \frac{\pi}{2}(\varphi,|H| \varphi)_{L^{2}}, \forall \varphi \in H^{1 / 2}\left(\mathbb{R}^{3}, \mathbb{C}^{4}\right) \\
(\varphi, W(x) \varphi)_{L^{2}} & \leq \frac{1}{2}\left(\frac{\pi}{2}+\frac{2}{\pi}\right)(\varphi,|H| \varphi)_{L^{2}}, \forall \varphi \in \Lambda^{+}\left(H^{1 / 2}\right) \cup \Lambda^{-}\left(H^{1 / 2}\right) \\
\|W(x) \varphi\|_{L^{2}} & \leq 2\|\nabla \varphi\|_{L^{2}} \leq 2\|H \varphi\|_{L^{2}}, \quad \forall \varphi \in H^{1}\left(\mathbb{R}^{3}, \mathbb{C}^{4}\right)
\end{aligned}
$$

Remark. Equalities (2.3) are a consequence of the following fact : in Fourier variables, the projectors $\Lambda^{ \pm}$are multiplication operators given by the following expressions:

$$
\hat{\Lambda}^{ \pm}(\xi)=\frac{ \pm \hat{H}+\sqrt{|\xi|^{2}+1}}{2 \sqrt{|\xi|^{2}+1}}
$$

Inequalities (P2) are classical, except for (2.5), whose proof, based on a method of Evans-Perry-Siedentop [16], is contained in the recent papers [8], [32] and [33]. For the proof of (2.4), see [19] and [21].

Part (P1) of the above lemma enables us to decompose the space $Y=H^{1 / 2}\left(\mathbb{R}^{3}, \mathbb{C}^{4}\right)$ as the direct sum of two infinite dimensional Hilbert spaces $Y^{ \pm}$which are stable under the action of $H$. Furthermore, a norm in $Y$ is given by

$$
\|\varphi\|_{Y}=\left(\varphi,\left(H \Lambda^{+}-H \Lambda^{-}\right) \varphi\right)_{Y \times Y^{\prime}}^{1 / 2}=(\varphi,|H| \varphi)^{1 / 2}
$$


This norm is the usual $H^{1 / 2}$-norm and for $\|.\|_{Y}, Y^{+}$and $Y^{-}$are orthogonal, as well as for $\|.\|_{L^{2}}$. In the sequel, $\|$.$\| will denote the L^{2}\left(\mathbb{R}^{3}, \mathbb{C}^{4}\right)$-norm and $\|.\|_{Y}$ the $H^{1 / 2}$-norm defined in (2.8).

Based on Lemma 2.1, we define now the class of scalar potentials $V$ to be considered all throughout this section. In the following, we will need to introduce the Marcinkiewicz space $M^{3}\left(\mathbb{R}^{3}\right)$ (see [2]).

Definition 2.2 . We say that a scalar potential $V$ defined a.e. in $\mathbb{R}^{3}$ satisfies assumption (H1) if it belongs to $M^{3}\left(\mathbb{R}^{3}\right)+L^{\infty}\left(\mathbb{R}^{3}\right)$ and if there is a positive constant $\delta$ such that

$$
\Lambda^{+}(H+V) \Lambda^{+} \geq \delta \sqrt{1-\Delta}, \quad \Lambda^{-}(H+V) \Lambda^{-} \leq-\delta \sqrt{1-\Delta} \text { in } Y .
$$

Remark 1. If $V$ belongs to $M^{3}\left(\mathbb{R}^{3}\right)+L^{\infty}\left(\mathbb{R}^{3}\right)$, then $V$ is $|H|$-bounded. In particular, any $|V| \leq a|x|^{-\beta}+C$ is $|H|$-bounded for all $a, C>0, \beta \in(0,1]$. If $|V| \leq a|x|^{-1}$, then, by (2.5), $V$ satisfies (H1) if and only if $a<(\pi / 4+$ $1 / \pi)^{-1} \approx 0.9$. Moreover, any $V \in L^{\infty}\left(\mathbb{R}^{3}\right)$ satisfies (H1) if $\|V\|_{\infty}<1$.

Remark 2. Assumption (H1) implies that for all constants $\kappa>1$ close to 1 , there is a positive constant $\delta(\kappa)>0$ such that :

$$
\Lambda^{+}(H+\kappa V) \Lambda^{+} \geq \delta(\kappa) \Lambda^{+}, \quad \Lambda^{-}(H+\kappa V) \Lambda^{-} \leq-\delta(\kappa) \Lambda^{-} \quad \text { on } \quad Y,
$$

In the sequel, we consider potentials $V$ satisfying (H1) and for those, we will look for eigenvalues of $H+V$ in the spectral gap of $H$. Before starting this program, let us recall some well-known results on the spectrum of $(H+V)$. For precise references and proofs, see [31].

Remark 3. Using the Friedrichs extension theorem, inequality (2.9) implies that $\Lambda^{+}(H+V) \Lambda^{+}$(resp. $\left.\Lambda^{-}(H+V) \Lambda^{-}\right)$can be defined, in a unique way, as a self-adjoint operator with domain contained in $Y^{+}$(resp. $Y^{-}$).

Theorem 2.3 (see e.g. [31]; Theorems 4.5, 4.6, 4.7) Let $V$ be a scalar potential which is $H$-bounded and such that $V(x) \rightarrow 0$ as $|x| \rightarrow+\infty$ and the operator $H+V$ is essentially self-adjoint. Then

$$
\sigma_{\text {ess }}(H+V)=\sigma_{\text {ess }}(H) .
$$

Eigenvalues belonging to the essential spectrum are not stable. So, stable Dirac states will be represented by eigenvalues lying in the gap of the 
essential spectrum of $H$, i.e. in the interval $(-1,1)$. There are a number of results concerning the nonexistence of eigenvalues lying in the essential spectrum of Dirac operators. Let us mention the work of A. Berthier and $\mathrm{V}$. Georgescu (see $[4,5]$ ), where it is proven that if the scalar potential $V$ satisfies very general decay conditions at infinity, there is no eigenvalue of the operator $H+V$ in the essential spectrum. In the particular case of the Coulomb potential, an elementary method based on the virial theorem implies the same result (see [31]). There are also results about the number of eigenvalues lying in the gap of the essential spectrum of $H$. More precisely, these results give conditions for the existence of an infinity of eigenvalues in the gap $(-1,1)$. In the case of only a finite number of eigenvalues in this interval, there are estimates about their number. For more details, see the exhaustive reference list in [31].

\section{3 - Minimax characterization of Dirac eigenvalues}

This section is devoted to the proof of a minimax characterization for the positive eigenvalues of $H+V$. More precisely, let us define the nondecreasing sequence $\left\{\lambda_{k}\right\}$ by

$$
\lambda_{k}(V)=\inf _{\substack{F \subset Y^{+} \\ \text {vector space } \\ \text { dim } F=k}} \sup _{\substack{\varphi \in F \oplus Y^{-} \\ \varphi \neq 0}} \frac{((H+V) \varphi, \varphi)}{(\varphi, \varphi)} .
$$

Then, we have the following :

Theorem 3.1. Let $V$ be a scalar potential satisfying assumption (H1). Assume also that $V \in L^{\infty}\left(\mathbb{R}^{3} \backslash \bar{B}_{R_{0}}\right)$ for some $R_{0}>0$ and that we have :

$$
\lim _{R \rightarrow+\infty}\|V\|_{L^{\infty}(|x|>R)}=0, \quad \lim _{R \rightarrow+\infty} \sup \operatorname{ess}_{|x|>R} V(x)|x|^{2}=-\infty .
$$

Then, $\lambda_{k}$ is an eigenvalue of $H+V,\left\{\lambda_{k}\right\}_{k \geq 1}$ is the non-decreasing sequence of eigenvalues of $H+V$ in the interval $[0,1)$, counted with multiplicity, and

$$
0<\delta \leq \lambda_{1} \leq \lambda_{k}<1, \quad \lim _{k \rightarrow+\infty} \lambda_{k}=1
$$

Remark. As it is clear from (3.1), all the eigenfunctions of $H+V$ corresponding to the eigenvalues $\lambda_{k}$ have Morse index equal to $+\infty$ as critical points of the Rayleigh quotient.

The proof of Theorem 3.1 will be carried out after we prove some technical auxiliary results. 
Lemma 3.2. Let $V$ satisfy (H1). Assume that there are at least $k$ eigenvalues of $H+V$ in $(0,1)$, counted with multiplicity, $\mu_{1} \leq \cdots \leq \mu_{k}$. Then, $\lambda_{k} \leq \mu_{k}$.

\section{Proof.}

Let $e_{1}, \cdots, e_{k}$ be an orthonormal family of eigenvectors, associated to the eigenvalues $\mu_{1}, \cdots, \mu_{k}$. Let $F=\operatorname{span}\left(\Lambda^{+} e_{1}, \cdots, \Lambda^{+} e_{k}\right)$. This space has dimension $k$, otherwise there would be a linear combination $\varphi$ of $e_{1}, \cdots, e_{k}$ with $\varphi \in Y^{-}$, hence $(\varphi,(H+V) \varphi)<0$ by $(\mathrm{H} 1)$ : this is impossible, since $\mu_{k} \geq \cdots \geq \mu_{1} \geq 0$. If $\Pi$ denotes the projector on $F+Y^{-}$, then the operator $-\Pi(H+V) \Pi$ is self-adjoint and bounded below, and its smallest eigenvalue is $-\mu_{k}$, by the standard theory of Rayleigh quotients (see [29], vol 4). This theory also gives

$$
\mu_{k}=\sup _{\substack{\varphi \in F \oplus Y^{-} \\ \varphi \neq 0}} \frac{((H+V) \varphi, \varphi)}{(\varphi, \varphi)}
$$

hence $\mu_{k} \geq \lambda_{k}$.

Remark. Let $S$ be the $4 \times 4$ matrix $\left(\begin{array}{cc}0 & -i \\ i & 0\end{array}\right)$. $S$ is a self-adjoint involution which anticommutes with $H$. So, if $\psi$ is an eigenvector of $H+V$ with associated eigenvalue $\lambda$, then $S \psi$ is an eigenvector of $H-V$ with eigenvalue $-\lambda$. As a consequence, Lemma 3.2 implies the following result:

Corollary 3.3. Let $V$ satisfy (H1). Then, there is no eigenvalue of $H+V$ in the interval $\left(-\lambda_{1}(-V), \lambda_{1}(V)\right)$. If, moreover, $V \leq 0$ a.e., then, $-\lambda_{1}(-V)=$ -1 and there is no eigenvalue of $H+V$ in the interval $\left(-1, \lambda_{1}(V)\right)$.

Let us now begin the study of the non-decreasing sequence $\left\{\lambda_{k}\right\}$ defined by (3.1). It is straightforward to see that $\lambda_{1} \geq \delta>0$, from (H1). The next lemma tells us that the supremum $\sup _{\substack{\varphi \in F \oplus Y^{-} \\ \varphi \neq 0}} \frac{((H+V) \varphi, \varphi)}{(\varphi, \varphi)}$ is in fact a maximum.

Lemma 3.4. Let $V$ satisfy (H1). Then, for all $k \geq 1$, and $F$ a $k$ dimensional subspace of $Y^{+}$, there is an orthonormal family $\varphi_{1}, \cdots, \varphi_{k}$ of eigenvectors of $\Pi(H+V) \Pi$ in $F+Y^{-}$, with eigenvalues $\gamma_{k} \geq \cdots \geq \gamma_{1}>0$ ( $\Pi$ is the projector on $\left.F+Y^{-}\right)$. Moreover, $(\psi, \Pi(H+V) \Pi \psi) \leq 0$ for any $\psi$ in the orthogonal space of $\operatorname{span}\left(\varphi_{1}, \cdots, \varphi_{k}\right)$ for the $L^{2}$ scalar product in 
$F+Y^{-}$and

$$
\gamma_{k}=\sup _{\substack{\varphi \in F \oplus Y^{-} \\ \varphi \neq 0}} \frac{((H+V) \varphi, \varphi)}{(\varphi, \varphi)}
$$

so $\varphi_{k}$ is a maximum point.

Proof. Once again, this follows immediately from the standard theory of Rayleigh quotients (see [29], vol 4), applied to the semibounded self-adjoint operator $-\Pi(H+V) \Pi$ in the Hilbert space $F+Y^{-}$, defined as a Friedrichs extension of the corresponding minimal operator.

Now we proceed to obtain upper bounds for the $\lambda_{k}$ 's. This will be crucial to prove that the $\lambda_{k}$ 's are achieved.

Lemma 3.5. Let $V$ a scalar potential satisfying (H1)-(H2). Then, for all $k \geq 1$, there exists a $k$-dimensional subspace of $Y^{+}, F_{k}$, such that

$$
\sup _{\substack{\varphi \in F_{k} \oplus Y^{-} \\ \varphi \neq 0}} \frac{((H+V) \varphi, \varphi)}{(\varphi, \varphi)}<1
$$

Corollary 3.6. If $V$ satisfies (H1)-(H2), then for all $k \geq 1$,

$$
\lambda_{k}<1
$$

The proof of the corollary is immediate.

Proof of Lemma 3.5. Let $G$ be the Hilbert subspace of $Y$ of the functions $e(x)$ whose Fourier transform can be written as $\hat{e}(\xi)=\theta(|\xi|)\left(\begin{array}{l}1 \\ 0 \\ 0 \\ 0\end{array}\right)$, $\theta \in C^{\infty}([0,1], \mathbb{R})$, supp $\theta \subset(0,1)$.

We note that for such functions $e,\left(\alpha_{k} \partial_{k} e, e\right)=0$. Let us now choose any $e_{1} \in G$ such that $\left\|e_{1}\right\|=1$. We define $e_{\lambda}(x)=\lambda^{-3 / 2} e_{1}\left(\frac{x}{\lambda}\right)$ for $\lambda>0$. Consider now any $\varphi^{-} \in Y^{-}$satisfying $\left\|\varphi^{-}\right\| \leq 1$ and take $\mu=\mu(\lambda) \geq 0$ such that $\left\|\varphi^{-}+\mu e_{\lambda}\right\|=1$. From the properties of $G$ and of the operator $H$, we have

$$
\left\{\begin{aligned}
A_{\lambda}\left(e_{1}\right):= & \left((H+V)\left(\varphi^{-}+\mu e_{\lambda}\right), \varphi^{-}+\mu e_{\lambda}\right) \\
= & \mu^{2}\left(\int_{\mathbb{R}^{3}}\left|e_{1}(x)\right|^{2} d x+\int_{\mathbb{R}^{3}} V(\lambda x)\left|e_{1}(x)\right|^{2} d x\right) \\
& +\left((H+V) \varphi^{-}, \varphi^{-}\right)+2 \mu \operatorname{Re}\left((H+V) e_{\lambda}, \varphi^{-}\right)
\end{aligned}\right.
$$

Now, from (H1) we find that

$$
\left((H+V) \varphi^{-}, \varphi^{-}\right) \leq-\delta\left\|\varphi^{-}\right\|^{2}, \text { for some } \delta>0 .
$$


Moreover, by applying Cauchy-Schwarz inequality,

$$
\begin{aligned}
\left|\left(H e_{\lambda}, \varphi^{-}\right)\right| & \leq \lambda^{-1}|| \vec{\alpha} \cdot \vec{\nabla} e_{1}\|\| \varphi^{-}\|+\| \Lambda^{-} e_{\lambda}\|\| \varphi^{-} \|, \\
\left|\left(V e_{\lambda}, \varphi^{-}\right)\right| & \leq\left(\int|V(\lambda x)|^{2}\left|e_{1}(x)\right|^{2} d x\right)^{1 / 2}\left\|\varphi^{-}\right\|
\end{aligned}
$$

Now, let us prove that $\left\|\Lambda^{-} e_{\lambda}\right\|=O\left(\lambda^{-1}\right)$. Indeed,

$$
\left\|\Lambda^{-} e_{\lambda}\right\|_{L^{2}}^{2}=\left(\Lambda^{-} e_{\lambda}, \Lambda^{-} e_{\lambda}\right)=\left(\Lambda^{-} e_{\lambda}, e_{\lambda}\right),
$$

since $\Lambda^{-}$is a projection operator. By $(2.7)$,

$$
\begin{aligned}
& \left\|\Lambda^{-} e_{\lambda}\right\|_{L^{2}}^{2}=\int_{\mathbb{R}^{3}} \frac{\sqrt{1+|\xi|^{2}}-1}{2 \sqrt{1+|\xi|^{2}}}\left|\hat{e}_{\lambda}(\xi)\right|^{2} d \xi \\
& \quad \leq \frac{1}{4} \int_{\mathbb{R}^{3}}|\xi|^{2}\left|\hat{e}_{\lambda}(\xi)\right|^{2} d \xi=\frac{1}{4 \lambda^{2}} \int_{\mathbb{R}^{3}}|\xi|^{2}\left|\hat{e}_{+}(\xi)\right|^{2} d \xi
\end{aligned}
$$

Thus, $\left\|\Lambda^{-} e_{\lambda}\right\|_{L^{2}}^{2} \leq \frac{C_{1}^{2}}{\lambda^{2}}$ with $C_{1}^{2}=\frac{1}{4} \int_{\mathbb{R}^{3}}|\xi|^{2}\left|\hat{e}_{+}(\xi)\right|^{2} d \xi$.

Moreover, we can use assumption (H2) to obtain

$$
\begin{gathered}
\varlimsup_{\lambda \rightarrow+\infty} \lambda^{2} \int_{\mathbb{R}^{3}} V(\lambda x)\left|e_{1}(x)\right|^{2} d x=-\infty \\
\int_{\mathbb{R}^{3}}|V(\lambda x)|^{2}\left|e_{1}(x)\right|^{2} d x \leq \frac{\delta}{4} \int_{\mathbb{R}^{3}}|V(\lambda x)|\left|e_{1}(x)\right|^{2} d x,
\end{gathered}
$$

for $\lambda$ large enough. Now, for $e_{1}$ and $\varphi^{-}$given, $\mu$ depends on $\lambda$. Indeed, $\left\|\varphi^{-}+\mu e_{\lambda}\right\|=1$ implies that $\mu\left\|\Lambda^{+} e_{\lambda}\right\| \leq 1$ and again from (2.7), it is easy to see that for $\lambda$ large,

$$
\left\|\Lambda^{+} e_{\lambda}\right\|_{L^{2}}^{2}=1-\left\|\Lambda^{-} e_{\lambda}\right\|_{L^{2}}^{2} \geq 1-\frac{C_{1}^{2}}{\lambda^{2}}, \quad \text { i.e., } \mu \leq 1+\frac{C_{1}^{2}}{2 \lambda^{2}},
$$

Estimates (3.5) to (3.11) and an optimization w.r.t. $\left\|\varphi^{-}\right\|$imply that

$$
\begin{aligned}
& A_{\lambda}\left(e_{1}\right) \leq \mu^{2}\left(1+\int_{\mathbb{R}^{3}} V(\lambda x)\left|e_{1}(x)\right|^{2} d x\right)-\delta\left\|\varphi^{-}\right\|^{2} \\
& \quad+2 \mu\left(\frac{\left\|\vec{\alpha} \cdot \vec{\nabla} e_{1}\right\|}{\lambda}+\frac{C_{1}}{\lambda}+\left(\int|V(\lambda x)|^{2}\left|e_{1}(x)\right|^{2} d x\right)^{1 / 2}\right)|| \varphi^{-} \| \\
& \quad \leq \mu^{2}\left(1+\int_{\mathbb{R}^{3}} V(\lambda x)\left|e_{1}(x)\right|^{2} d x+\frac{2}{\delta} \int_{\mathbb{R}^{3}}|V(\lambda x)|^{2}\left|e_{1}(x)\right|^{2} d x+O\left(\frac{1}{\lambda^{2}}\right)\right) \\
& \quad \leq 1+O\left(\frac{1}{\lambda^{2}}\right)+\frac{1}{2} \int_{\mathbb{R}^{3}} V(\lambda x)\left|e_{1}(x)\right|^{2} d x<1 \quad \text { for } \lambda \text { large } .
\end{aligned}
$$


To end the proof, we consider a $k$-dimensional subspace of $G, G_{k}$ and define $F_{k}^{\lambda}$ as

$$
F_{k}^{\lambda}=\left\{\lambda^{-\frac{3}{2}} e_{1}\left(\frac{\dot{\lambda}}{\lambda}\right) \mid e_{1} \in G_{k}\right\} .
$$

Inequality (3.12) can be made uniform for $e_{1} \in G_{k}$ in such a way that there exists $A_{\lambda}>0$ such that

$$
A_{\lambda}\left(e_{1}\right)_{\lambda \sim+\infty}^{<} 1 \text { uniformly for all } e_{1} \in G_{k}, \lambda>0 \text { large. }
$$

Then, it is sufficient to define

$$
F_{k}:=F_{k}^{\lambda}, \quad \lambda \text { large }
$$

and the lemma is proved.

\section{Proof of Theorem 3.1.}

From Lemma 3.4, the supremum appearing in the definition of $\lambda_{k}$ is achieved for every $k$-dimensional subspace $F$ of $Y^{+}$.

Let us now prove that the min-max is also achieved. We begin with the case $k=1$. Let us consider a minimizing sequence $\left\{\varphi_{1}^{n}\right\}$ such that $\left\|\varphi_{1, n}\right\|=1, \Lambda^{+} \varphi_{1, n} \neq 0$,

$$
\lambda_{1, n}:=\sup _{\substack{\varphi \in \mathbb{C} \varphi_{1, n}+Y^{-} \\ \varphi \neq 0}} \frac{((H+V) \varphi, \varphi)}{(\varphi, \varphi)},
$$

is achieved by $\varphi_{1, n}$ and

$$
\lim _{n} \lambda_{1, n}=\lambda_{1}
$$

The Euler-Lagrange equation satisfied by $\varphi_{1, n}$ reads

$$
(H+V) \varphi_{1, n}=\lambda_{1, n} \varphi_{1, n}+f_{n},
$$

for some $f_{n}$ which is orthogonal to $Y^{-} \oplus \mathbb{C} \varphi_{1, n}$ for the duality product in $H^{-1 / 2} \times H^{1 / 2}$. Multiplying both sides of this equation by $\varphi_{1, n}^{+}-\varphi_{1, n}^{-}$, where $\varphi_{1, n}^{ \pm}:=\Lambda^{ \pm} \varphi_{1, n}$ we obtain

$$
\left((H+V) \varphi_{1, n}^{+}, \varphi_{1, n}^{+}\right)-\left((H+V) \varphi_{1, n}^{-}, \varphi_{1, n}^{-}\right) \leq \lambda_{1, n} .
$$

Hence, by hypothesis (H1), the sequence $\left\{\varphi_{1, n}\right\}_{n}$ is uniformly bounded in $Y$ and therefore, relatively compact for the $Y$-weak and $L_{\text {loc }}^{q}\left(\mathbb{R}^{3}\right)$-strong topologies, $1 \leq q<3$. Up to subsequences, we may assume that $\varphi_{1} \in Y$ is the limit of $\left\{\varphi_{1, n}\right\}_{n}$ in those topologies. Finally, assume that the sequence 
of Lagrange multipliers $\left\{f_{n}\right\}$ converges to 0 in $H^{-1 / 2}\left(\mathbb{R}^{3}\right)$. If this holds, we have

$$
(H+V) \varphi_{1}=\lambda_{1} \varphi_{1}
$$

Thus, under the above assumption on $f_{n}$, Theorem 3.1 will be proved (in the case $k=1$ ) if we show that $\varphi_{1} \not \equiv 0$. Assume the contrary. Since $V_{|x| \rightarrow+\infty} 0$ and $V \in L_{\text {loc }}^{r}\left(\mathbb{R}^{3}\right)$ for $r \in(2,3), V \varphi_{1, n} \underset{n \rightarrow+\infty}{\longrightarrow} 0$ in $L^{2}\left(\mathbb{R}^{3}\right)+L^{s}\left(\mathbb{R}^{3}\right)$ for $s \in\left(\frac{6}{5}, \frac{3}{2}\right)$. But then, for $n$ large we can write

$$
\varphi_{1, n}=\left(H-\lambda_{1}^{n}\right)^{-1}\left(f_{n}-V \varphi_{1, n}\right)
$$

The operators $\left(H-\lambda_{1}^{n}\right)^{-1}$ are uniformly bounded, since $\lambda_{1} \in(0,1)$. So, if $f_{n} \underset{n}{\rightarrow} 0$ in $H^{-1 / 2}\left(\mathbb{R}^{3}\right)$ and $\varphi_{1} \equiv 0$,

$$
\varphi_{1, n} \underset{n \rightarrow+\infty}{\longrightarrow} 0 \text { in } L^{2}\left(\mathbb{R}^{3}\right)+H^{1}\left(\mathbb{R}^{3}\right)+W^{1, s}\left(\mathbb{R}^{3}\right),
$$

which contradicts the normalization $\left\|\varphi_{1, n}\right\|=1$.

Let us finally prove that the sequence $\left\{f_{n}\right\}$ has to converge to 0 in $H^{-1 / 2}\left(\mathbb{R}^{3}\right)$. If this were not the case, up to subsequences, we would find $\chi_{n} \in Y^{+},\left(\chi_{n}, \varphi_{1, n}\right)=0$ such that $\left\|\chi_{n}\right\|_{Y}=1$ and

$$
\left.\operatorname{Re}\left((H+V) \varphi_{1, n}, \chi_{n}\right)\right)=\operatorname{Re}\left(f_{n}, \chi_{n}\right) \geq \sigma>0
$$

for some $\sigma$ independent of $n$. Since $V$ is $|H|$-bounded, there exists a constant $C_{1}$ such that for all $n,\left|\left((H+V) \chi_{n}, \chi_{n}\right)\right| \leq C_{1}$. Then, for any $t>0$, let us consider the space $G_{t}=\operatorname{span}\left\{\varphi_{1, n}^{+}-t \chi_{n}\right\} \oplus Y^{-}$. Let us now take any $\psi \in G_{t}$ of the form $\psi=\varphi_{1, n}-t \chi_{n}+\varphi^{-}, \varphi^{-} \in Y^{-}$. We obtain :

$$
\begin{aligned}
((H+V) \psi, \psi)= & \lambda_{1}^{n}\left\|\varphi_{n}\right\|^{2}+2 \lambda_{1}^{n} \operatorname{Re}\left(\varphi_{n}, \varphi^{-}\right) \\
& +\left((H+V) \varphi^{-}, \varphi^{-}\right)-2 t \operatorname{Re}\left(f_{n}, \chi_{n}\right) \\
& +t^{2}\left((H+V) \chi_{n}, \chi_{n}\right)-2 t \operatorname{Re}\left((H+V) \varphi^{-}, \chi_{n}\right)
\end{aligned}
$$

Since $V$ satisfies assumption $(\mathrm{H} 1),((H+V) \psi, \psi) \leq 0$ for $\left\|\varphi^{-}\right\|_{Y}$ large. Hence, there exists a constant $M$ such that $((H+V) \psi, \psi) \geq 0$ implies $\left\|\varphi^{-}\right\|_{Y} \leq M$. This, (3.20) and our assumptions imply that for $\left\|\varphi^{-}\right\|_{Y} \leq M$,

$$
\begin{aligned}
((H+V) \psi, \psi) \leq \lambda_{1, n}\left\|\varphi_{1, n}\right\|^{2}+2 \lambda_{1, n} \operatorname{Re}\left(\varphi_{1, n}, \varphi^{-}\right) \\
\quad-\delta\left\|\varphi^{-}\right\|_{Y}^{2}-2 t \sigma+C_{1} t^{2}+2 C t\left\|\varphi^{-}\right\|_{Y} \\
\leq \lambda_{1, n}\left\|\varphi_{1, n}-t \chi_{n}+\varphi^{-}\right\|^{2}-2 t \sigma+C_{1} t^{2}+2 C t\left\|\varphi^{-}\right\|{ }_{Y}-\delta\left\|\varphi^{-}\right\|_{Y}^{2}
\end{aligned}
$$


with $C$ independent of $n$. Then, we optimize the above inequality in $\left\|\varphi^{-}\right\|_{Y}$ to obtain

$$
((H+V) \psi, \psi) \leq \lambda_{1, n}\left\|\varphi_{1, n}-t \chi_{n}+\varphi^{-}\right\|^{2}-2 t \sigma+\left(C_{1}+\frac{C^{2}}{\delta}\right) t^{2}
$$

and hence, for $t$ small enough,

$$
\sup _{\psi \in G_{t}} \frac{((H+V) \psi, \psi)}{(\psi, \psi)} \leq \lambda_{1, n}-\frac{t \sigma}{\|\psi\|^{2}}<\lambda_{1}-\varepsilon
$$

for some $\varepsilon>0$, because $\|\psi\|$ is uniformly bounded. Indeed, in the second step of the proof, we obtained an estimate for $\left\{\varphi_{1, n}\right\}$ in $Y$ and by assumption, $\left\|\varphi^{-}\right\| \leq M,\left\|\chi_{n}\right\| \leq 1$. The contradiction in (3.22) ends the proof in the case $k=1$.

Case $k>1$ : Assume that we have already solved the min-max problem corresponding to $\lambda_{i}, 1 \leq i \leq k-1$ and let $\varphi_{i}$ be the corresponding eigenfunction. If $F_{n}$ is a minimizing sequence for $\lambda_{k}$ and $\varphi_{k, n}$ the corresponding maximizer given by Lemma 3.4, we normalize it to have $\left\|\varphi_{k, n}\right\|=1$ and as above, the Euler-Lagrange equation satisfied by $\varphi_{k, n}$ is

$$
(H+V) \varphi_{k, n}=\lambda_{k, n} \varphi_{k, n}+f_{n},
$$

for some $f_{n}$ which is orthogonal to $Y^{-} \oplus F_{n}$ for the duality product in $H^{-1 / 2} \times H^{1 / 2}$ and

$$
\lim _{n} \lambda_{k, n}=\lambda_{k}
$$

If $\lambda_{k-1}<\lambda_{k}$, then we follow the same steps as in the case $k=1$ to conclude that the weak limit in $Y$ of $\varphi_{k, n}$ is a function $\varphi_{k}$ satisfying

$$
(H+V) \varphi_{k}=\lambda_{k} \varphi_{k}, \quad \varphi_{k} \not \equiv 0,
$$

and therefore, $\varphi_{k}$ is a solution for the min-max problem defining $\lambda_{k}$. Note that the only important change that we make here is to replace $G_{t}$ with a similar subspace $\tilde{G}_{t}:=\operatorname{span}\left\{\varphi_{1, n}, \ldots, \varphi_{k, n}-t \chi_{n}\right\}$.

If, on the contrary, $\lambda_{k-1}=\lambda_{k}$, let us then define $\ell$ as the largest integer in the set $\{1, \ldots, k\}$ such that $\lambda_{\ell}<\lambda_{k}$. Hence, $\lambda_{\ell+1}=\lambda_{k-1}=\lambda_{k}$ and we can find a subspace $G_{n}$ of $F_{n} \cap\left\{\varphi_{\ell+1}, \ldots, \varphi_{k-1}\right\}^{\perp}$, of dimension $\ell+1$, which is minimizing for $\lambda_{\ell+1}$. So, as in the preceding case, we find a function $\varphi$, solution of

$$
(H+V) \varphi=\lambda_{\ell+1} \varphi=\lambda_{k} \varphi, \quad \varphi \not \equiv 0,
$$


and by construction, $\left(\varphi, \varphi_{i}\right)=0$ for all $i \in\{\ell+1, \ldots, k-1\}$. We define $\varphi_{k}:=\varphi$, and by induction, this ends the proof.

\section{4 - A minimization procedure providing an eigenstate of Dirac operators : the ground state.}

In this section, we introduce a minimization method which, under appropriate assumptions on the potential $V$, yields the eigenvalues of $H+V$ lying in the spectral gap as well as the corresponding eigenfunctions.

The Dirac equation is originally written for 4-spinors. Here, we begin by making a reduction to an equivalent equation for 2 -spinors. Of course, this requires some conditions on $V$.

For any $\psi$ with values in $\mathbb{C}^{4}$, let us write $\psi=\left(\begin{array}{l}\varphi \\ \chi\end{array}\right)$, with $\varphi, \chi$ taking values in $\mathbb{C}^{2}$. Then, the equation

$$
(H+V) \psi=\lambda \psi \quad \text { in } \mathbb{R}^{3}
$$

is equivalent to the system

$$
\left\{\begin{array}{l}
L \chi=(E-V) \varphi \text { in } \mathbb{R}^{3} \\
L \varphi=(E+2-V) \chi
\end{array}\right.
$$

with $E=\lambda-1$ and $L=i(\vec{\sigma} \cdot \vec{\nabla})=\sum_{k=1}^{3} i \sigma_{k} \partial_{k}$.

If $V \leq 0$ a.e. in $\mathbb{R}^{3}$ and $E>-2$ (which is the case for stable eigenvalues, see Section 2), the system (4.2) can be written as

$$
L\left(\frac{L \varphi}{g_{E}}\right)+V \varphi=E \varphi, \quad \chi=\frac{L \varphi}{g_{E}} \quad \text { in } \mathbb{R}^{3}
$$

where $g_{E}=E+2-V$. Now, we make the following change of function :

$$
\varphi=\sqrt{g_{E}} \phi
$$

which is well defined (for $V \leq 0$ and $E>-2$ ). From (4.3) we obtain

$$
H_{E} \phi:=\sqrt{g_{E}} L\left(\frac{1}{g_{E}} L\left(\sqrt{g_{E}} \phi\right)\right)=(E-V)(E+2-V) \phi \text { in } \mathbb{R}^{3}
$$

and we notice that the operator acting on $\phi$ in the left-hand side of (4.4) is formally symmetric. If $\phi$ is a solution of (4.4), we have

$$
(\phi, \phi) E^{2}+2(\phi,(1-V) \phi) E-(\phi,(2-V) V \phi)-\left(\phi, H_{E} \phi\right)=0
$$


and we notice that (4.5) holds if and only if

$$
E=J^{ \pm}(E, \phi):=\frac{1}{(\phi, \phi)}( \pm \sqrt{\Delta(E, \phi)}-(\phi,(1-V) \phi))
$$

where $\Delta(E, \phi):=|(\phi, V \phi)|^{2}+(\phi, \phi)\left[(\phi, \phi)+\left(\phi, H_{E} \phi\right)-\left(\phi, V^{2} \phi\right)\right]$. Thus, we have obtained that if $E>-2$ is an eigenvalue of $\varphi \mapsto L\left(\frac{L \varphi}{g_{E}}\right)+V \varphi$, there exists an associated eigenfunction $\phi \not \equiv 0$ such that (4.6) holds. The main idea of Section 4 is to prove conversely that under appropriate conditions, critical points of $J^{+}(E, \phi)$ are solutions of equation (4.4). In fact, for a given potential $W$, we will prove this for the operators $H+a W$ with $a$ less than some constant $A_{W}$, depending only on the potential $W$.

In order to proceed further, let us define

$T(E, \phi):=(\phi, \phi)+\left(\phi, H_{E} \phi\right)-\left(\phi, V^{2} \phi\right)=\tilde{T}(E, \varphi):=\int_{\mathbb{R}^{3}} \frac{|L \varphi|^{2}+|\varphi|^{2}-V^{2}|\varphi|^{2}}{g_{E}}$ so that $\Delta(E, \phi)=(\phi, V \phi)^{2}+(\phi, \phi) T(E, \phi)$, provided $\varphi=\sqrt{g_{E}} \phi$.

We notice that since $E$ has to be real, $\Delta(E, \phi)$ must be nonnegative for all the functions $\phi$ which we will be considering. Also, for $\phi \not \equiv 0$,

$$
T(E, \phi) \geq 0
$$

if and only if $\Delta(E, \phi) \geq|(\phi, V \phi)|^{2} \geq 0$. Then, if $\phi \not \equiv 0, J^{+}(E, \phi) \geq$ $\frac{|(\phi, V \phi)|+(\phi, V \phi)}{(\phi, \phi)}-1 \geq-1$ and $J^{-}(E, \phi) \leq \frac{(\phi, V \phi)-|(\phi, V \phi)|}{(\phi, \phi)}-1 \leq-1$, with equality if and only if $T(E, \phi)=0$.

Thus, if $V \not \equiv 0$ is a nonpositive potential and if (4.7) holds for all $(E, \phi)$ in some adapted set $X$, with strict inequality, the only functional yielding positive stable eigenvalues of $H+V$ is $J^{+}$.

In this section, we will prove that critical points of $J^{+}$in a well chosen but natural class of functions solve equation (4.4). At a formal level, critical points $(E, \phi)$ of the functional $J^{+}(E, \phi)$, satisfying the constraint $E=$ $J^{+}(E, \phi)$, are indeed solutions of (4.4) (for more details, see step 4 in the proof of Theorem 4.3 below). Moreover, under adequate assumptions on $V$, we will find the "ground state energy" of $H+V$ as the minimum value of $J^{+}$.

Let $X_{0}$ be defined by

$$
X_{0}:=\left\{\varphi \in L^{2}\left(\mathbb{R}^{3}\right) ; \int_{\mathbb{R}^{3}} \frac{|L \varphi|^{2}+|\varphi|^{2}}{1+|V|} d x<+\infty\right\}
$$


and

$$
X:=\left\{\phi \in L^{2}\left(\mathbb{R}^{3}\right) ; \varphi:=\sqrt{g_{E}} \phi \in X_{0} \text { for some } E \in(-1,0)\right\} .
$$

Note that for all $\phi \in X, E \in(-1,0)$,

$$
\||\phi|\|_{E}^{2}:=\left(H_{E} \phi, \phi\right)+(\phi, \phi)=\int_{\mathbb{R}^{3}} \frac{|L \varphi|^{2}+|\varphi|^{2}}{g_{E}} d x<+\infty
$$

Note also that if $V$ is a nonpositive scalar potential and $\psi=\left(\begin{array}{c}\varphi \\ \chi\end{array}\right) \in L^{2} \backslash\{0\}$ is an eigenfunction of $H+V$, associated with an eigenvalue $\lambda>-1$, then the corresponding $\phi$ is in $X$ whenever $V \chi^{2} \in L^{1}\left(\mathbb{R}^{3}\right)$. This last condition is automatically satisfied if $V$ satisfies assumption (H1) of Section 3.

Now, assume that the scalar potential $W$ satisfies

$$
\begin{gathered}
W \in L_{l o c}^{\infty}\left(\mathbb{R}^{3} \backslash\{0\}\right) \text { and either } W \in L^{\infty}\left(\mathbb{R}^{3}\right) \text { or } W \underset{|x| \rightarrow 0}{\longrightarrow}-\infty \\
W \leq 0 \text { a.e. in } \mathbb{R}^{3}, \lim _{R \rightarrow+\infty}\|W\|_{L^{\infty}(|x|>R)}=0,
\end{gathered}
$$

$$
M_{W}:=\min \left(\inf _{\varphi \in X_{0}} \frac{\int_{\mathbb{R}^{3}}|W|^{-1}\left(|L \varphi|^{2}+|\varphi|^{2}\right)}{\int_{\mathbb{R}^{3}}|W||\varphi|^{2}}, \inf _{\varphi \in X_{0}} \frac{\int_{\mathbb{R}^{3}}|\nabla \varphi|^{2}+|\varphi|^{2}}{\int_{\mathbb{R}^{3}} W^{2}|\varphi|^{2}}\right)>0 .
$$

Remarks. Assumptions (J1) to (J3) are invariant by multiplication by a positive constant, i.e. if $W$ satisfies (J1) to (J3), then the same holds for $V=a W$ for all $a>0$. However, assumption (H1) (stated in Section 3) for $V=a W$ may strongly depend on the value of $a$.

If $W_{\beta}=-|x|^{-\beta}, \beta \in(0,1]$, it is easy to check that $W_{\beta}$ satisfies assumptions (J1) to (J3). To do so, one has to use classical Hardy-type inequalities.

It is also possible to replace a single singularity of $V$ at the origin by several isolated singularities.

Let us now derive some implications of asumptions (J1)-(J3) which will be useful in the sequel of the paper.

Proposition 4.1. Let $W$ a potential satisfying (J1) to (J3). For any $a_{0}>0$, we have

$$
l\left(a_{0}\right):=\inf _{0<a<a_{0}} \inf _{(E, \varphi) \in(-1,0) \times X_{0}} \frac{\int_{\mathbb{R}^{3}} \frac{|L \varphi|^{2}+|\varphi|^{2}}{E+2+a|W|}}{\int_{\mathbb{R}^{3}} \frac{a W^{2}|\varphi|^{2}}{E+2+a|W|}}>0 .
$$




\section{Proof of Proposition 4.1.}

If $W$ is bounded, the proof immediately follows. If, on the contrary, $W$ tends to $-\infty$ at 0 , then, if $l\left(a_{0}\right)=0$, there must exist a sequence $\left\{\left(E_{n}, \phi_{n}\right)\right\} \in(-1,0) \times X$ and $a_{n} \in\left(0, a_{0}\right]$ such that

$$
\int_{\mathbb{R}^{3}} \frac{W^{2}\left|\varphi_{n}\right|^{2}}{E_{n}+2+a_{n}|W|}=1, \quad \frac{1}{a_{n}} \int_{\mathbb{R}^{3}} \frac{\left|L \varphi_{n}\right|^{2}+\left|\varphi_{n}\right|^{2}}{E_{n}+2+a_{n}|W|} \underset{n \rightarrow+\infty}{\longrightarrow} 0 .
$$

Let us choose $\beta>0$ such that $2 \leq|W|$ a.e. in the set $\left\{x \in \mathbb{R}^{3} ; 0<\right.$ $|x| \leq 2 \beta\}$ and define $\ell:=\max _{|x| \geq \beta}|W(x)|$. Finally, we consider a function $\xi \in C^{1}\left(\mathbb{R}^{3}\right)$, with values in the interval $[0,1]$, such that $\xi \equiv 1$ on the ball of radius $\beta, B_{\beta}$, supp $\xi \subset B_{2 \beta}$ and $\nabla \sqrt{1-\xi^{2}} \in L^{\infty}$. Then,

$$
\begin{aligned}
\int_{\mathbb{R}^{3}} \frac{\left|L \varphi_{n}\right|^{2}+\left|\varphi_{n}\right|^{2}}{E_{n}+2+a_{n}|W|} & \geq \int_{\mathbb{R}^{3}} \frac{\left|L\left(\xi \varphi_{n}\right)\right|^{2}+\left|\xi \varphi_{n}\right|^{2}}{\left(a_{n}+1\right)|W|}+ \\
& +\frac{\left|L\left(\sqrt{1-\xi^{2}} \varphi_{n}\right)\right|^{2}+\left(1-\xi^{2}\right)\left|\varphi_{n}\right|^{2}}{2+a_{n} \ell}+r_{n} a_{n}
\end{aligned}
$$

with $\lim _{n \rightarrow+\infty} r_{n}=0$. By using (J3) we find :

$$
\begin{aligned}
\int_{\mathbb{R}^{3}} \frac{\left|L \varphi_{n}\right|^{2}+\left|\varphi_{n}\right|^{2}}{E_{n}+2+a_{n}|W|} & \geq \frac{M_{W}}{a_{n}+1} \int_{\mathbb{R}^{3}}|W| \varphi_{n}^{2} \xi^{2}+M_{W} \int_{\mathbb{R}^{3}} \frac{W^{2} \varphi_{n}^{2}\left(1-\xi^{2}\right)}{2+a_{n} \ell}+r_{n} a_{n} \\
& \geq \frac{M_{W} a_{n}}{a_{n}+1} \int_{\mathbb{R}^{3}} \frac{W^{2} \varphi_{n}^{2} \xi^{2}}{E_{n}+2+a_{n}|W|}+\frac{M_{W}}{2+a_{n} \ell} \int_{\mathbb{R}^{3}} \frac{W^{2} \varphi_{n}^{2}\left(1-\xi^{2}\right)}{E_{n}+2+a_{n}|W|}+r_{n} a_{n} \\
& \geq M_{W} \min \left(\frac{a_{n}}{a_{n}+1}, \frac{1}{2+a_{n} \ell}\right)+r_{n} a_{n}
\end{aligned}
$$

and this shows that (4.9) cannot hold, what ends the proof.

A straightforward corollary of the above proposition is the following

Corollary 4.2. If a potential $W$ satisfies (J1) to (J3), then for $a>0$ small enough, $V=a W$ satisfies

$$
I_{V}:=\inf _{\substack{E, \varphi) \in(-1,0) \times X_{0} \\ J^{+}(E, \phi) \leq E}} \frac{\int_{\mathbb{R}^{3}} \frac{|L \varphi|^{2}+|\varphi|^{2}}{E+2+|V|}}{\int_{\mathbb{R}^{3}} \frac{V^{2}|\varphi|^{2}}{E+2+|V|}}>1 .
$$

Proof. Since the function $l$ is nonincreasing and stricly positive on $\mathbb{R}^{+}$, $l(a) / a>1$ for $a>0$ small enough. Finally, $I_{a W} \geq(l(a) / a)$, since the extra constraint $J^{+}(E, \phi) \leq E$ only increases the value of the infimum. 
Remark. Note that

$$
\begin{gathered}
I_{V}>1 \Rightarrow \int_{\mathbb{R}^{3}} \frac{V^{2}(x)|\varphi(x)|^{2}}{g_{E}(x)} d x<\int_{\mathbb{R}^{3}} \frac{|L \varphi|^{2}+|\varphi|^{2}}{g_{E}(x)} d x \\
\Leftrightarrow T(E, \phi)=\left(\phi, H_{E} \phi\right)+(\phi, \phi)-\left(\phi, V^{2} \phi\right)>0 \\
\Leftrightarrow J^{+}(E, \phi)>-1
\end{gathered}
$$

for all $\left(E, \phi=\frac{\varphi}{\sqrt{g_{E}}}\right) \in[-1,0] \times X$, such that $J^{+}(E, \phi) \leq E$.

In order to find the minimal "eigenvalue" $E$ of (4.4), we would like to minimize $J^{+}(E, \phi)$ over the set $\left\{(E, \phi) ; \phi \not \equiv 0, J^{+}(E, \phi)=E\right\}$. Let us introduce a relaxed minimization problem over the set

$$
D_{V}:=\left\{(E, \phi) \in(-2,0) \times X ; \phi \not \equiv 0, J^{+}(E, \phi) \leq E\right\} .
$$

Now we are ready to state the main theorem of this section.

Theorem 4.3. Assume that $V$ is a scalar potential satisfying (J1) to (J3) such that

$$
I_{V}>1
$$

and

$$
D_{V} \neq \emptyset
$$

Then, the following infimum

$$
E_{0}:=\inf \left\{E \in(-2,0) ; \exists \phi \in X \text { with }(E, \phi) \in D_{V}\right\}
$$

is achieved in $D_{V}$ by some $\left(E_{0}, \phi_{0}\right)$. Moreover,

$$
\phi_{0} \not \equiv 0, J^{+}\left(E_{0}, \phi_{0}\right)=E_{0} \in(-1,0)
$$

and $\phi \equiv \phi_{0}$ is a solution of (4.4) with $E=E_{0}$.

Remark. By Corollary 4.2 and Theorem 4.3, if $W$ satisfies (J1) to (J3) and $a>0$ is small enough, then the "ground state" of the operator $H+a W$ is given by the minimization of $J^{+}$over the set $D_{a W}$. So, Theorem 4.3 applies to $V=-a|x|^{-\beta}, \beta \in(0,1], a>0$ small. Moreover, as shown below (see Proposition 4.6), this theorem is optimal for the Coulomb potential: in the case $\beta=1$, one can take any $a<1$.

Corollary 4.4. Under the assumptions of Theorem 4.3, the function $\psi_{0}=$ $\left(\begin{array}{l}\varphi_{0} \\ \chi_{0}\end{array}\right)$, with $\varphi_{0}=\sqrt{g_{E_{0}}} \phi_{0}, \chi_{0}=L \varphi_{0} / g_{E_{0}}$ satisfies

$$
H \psi_{0}+V \psi_{0}=\left(E_{0}+1\right) \psi_{0} \quad \text { in } \quad \mathbb{R}^{3}, \psi_{0} \not \equiv 0
$$


Moreover, $E_{0}+1$ is the smallest eigenvalue of $(H+V)$ in $(0,1)$ corresponding to an eigenfunction $\psi=\left(\begin{array}{l}\varphi \\ \chi\end{array}\right)$ such that $\varphi \in X_{0}$.

Proof of Corollary 4.4. The first assertion follows immediately from Theorem 4.3 and the introduction of this section. As for the fact that $E_{0}+1$ is the smallest eigenvalue of $H+V$ in $(0,1)$ such that $\varphi \in X_{0}$, we just invoke the fact that $J^{-}$maps $X$ into $(-\infty,-1)$.

\section{Proof of Theorem 4.3.}

Step 1 : $E_{0} \in(-1,0)$.

Since the set $D_{V}$ is not empty, we have : $E_{0}<0$. Also, from the definition of $J^{+}$and (4.14), $E_{0} \geq-1$. Assume by contradiction that $E_{0}=$ -1 . Then, since the functional $J^{+}$is homogeneous of degree 0 , there exists a sequence $\left\{\left(E_{n}, \phi_{n}\right)\right\}$ in $D_{V}$ such that

$$
\int_{\mathbb{R}^{3}} h_{E_{n}}\left|\phi_{n}\right|^{2}=1, \quad-1<J^{+}\left(E_{n}, \phi_{n}\right) \leq E_{n} \underset{n \rightarrow+\infty}{\longrightarrow}-1,
$$

a contradiction with the following lemma :

Lemma 4.5. Assume that $V$ satisfies (J1)-(J3) and that $I_{V}>1$. If $E>-1$ and $\phi \in X$ satisfy

$$
\int_{\mathbb{R}^{3}}(E+1-V)|\phi|^{2} d x=1 \quad \text { (normalization condition) }
$$

and

$$
J^{+}(E, \phi) \leq E
$$

then,

$$
E+1>\min \left\{\sqrt{\frac{\zeta}{2(1+\zeta)}}, \frac{1}{\sqrt{1+\zeta}}, \frac{\zeta}{1+2 \sqrt{1+\zeta}+\sqrt{5+4(\zeta+\sqrt{1+\zeta})}}\right\}
$$

with $\zeta=I_{V}-1$.

\section{Proof of Lemma 4.5.}

By (ii), $\theta:=E+1 \geq J^{+}(E, \phi)+1=\|\phi\|^{-2}\left\{\sqrt{\Delta(E, \phi)}+\int_{\mathbb{R}^{3}} V|\phi|^{2} d x\right\}$. Now, combining (i) and (ii) we obtain

$$
\Delta(E, \phi)=\|\phi\|^{2} T(E, \phi)+(\phi, V \phi)^{2} \leq 1 .
$$


By (i) again,

$$
\|\phi\|^{2} T(E, \phi) \leq 1-\left(1-\theta\|\phi\|^{2}\right)^{2}
$$

and then

$$
T(E, \phi) \leq \theta\left(2-\theta\|\phi\|^{2}\right) \leq 2 \theta .
$$

On the other hand, since $I_{V}>1$, by definition of $\zeta$, we have

$$
\frac{\left(\phi, H_{E} \phi\right)+(\phi, \phi)}{1+\zeta} \geq\left(\phi, V^{2} \phi\right)
$$

Hence,

(4.23) $T(E, \phi)=\left(\phi, H_{E} \phi\right)+(\phi, \phi)-\left(\phi, V^{2} \phi\right) \geq \frac{\zeta}{1+\zeta}\left(\|\phi\|^{2}+\left(\phi, H_{E} \phi\right)\right)$,

and then

$$
\begin{gathered}
\|\phi\|^{2} \leq\left(\phi, H_{E} \phi\right)+\|\phi\|^{2} \leq \frac{(1+\zeta) T(E, \phi)}{\zeta}, \\
\int_{\mathbb{R}^{3}} g_{E}(x)|\phi|^{2} d x=1+\|\phi\|^{2} \leq 1+\frac{(1+\zeta) T(E, \phi)}{\zeta} .
\end{gathered}
$$

By using Cauchy-Schwarz's inequality and (4.25) we also have

$$
\left(\int_{\mathbb{R}^{3}} V|\phi|^{2} d x\right)^{2} \leq\left(1+\frac{(1+\zeta) T(E, \phi)}{\zeta}\right) \int_{\mathbb{R}^{3}} \frac{V^{2}|\phi|^{2}}{g_{E}} d x .
$$

Clearly, for any $a, b>0$, we have $\frac{a}{b} \geq I_{V}$ if and only if $b \leq \frac{a-b}{I_{V}-1}=$ $\zeta^{-1}(a-b)$. Taking $a=\int_{\mathbb{R}^{3}} \frac{|L \phi|^{2}+|\phi|^{2}}{g_{E}} d x$ and $b=\int_{\mathbb{R}^{3}} \frac{V^{2}|\phi|^{2}}{g_{E}} d x$, we have $a-b=T(E, \phi)$ and

$$
\left(\int_{\mathbb{R}^{3}} V|\phi|^{2} d x\right)^{2} \leq \frac{T(E, \phi)}{\zeta}\left(1+\frac{(1+\zeta) T(E, \phi)}{\zeta}\right)
$$

Using (4.24) and then (4.27) we have

$$
\begin{aligned}
\frac{\theta(1+\zeta)}{\zeta} T(E, \phi) & \geq \theta\|\phi\|^{2}=1+\int_{\mathbb{R}^{3}} V|\phi|^{2} d x \\
& \geq 1-\sqrt{\frac{T(E, \phi)}{\zeta}\left(1+\frac{1+\zeta}{\zeta} T(E, \phi)\right)}
\end{aligned}
$$

If $\theta \leq \sqrt{\frac{\zeta}{2(1+\zeta)}},\left(1-\frac{\theta(1+\zeta) T(E, \phi)}{\zeta}\right) \geq 1-\frac{2(1+\zeta) \theta^{2}}{\zeta} \geq 0$, because of (4.21), and then

$$
T(E, \phi)\left(1+\frac{(1+\zeta) T(E, \phi)}{\zeta}\right) \geq \zeta\left(1-\theta \frac{(1+\zeta)}{\zeta} T(E, \phi)\right)^{2}
$$


or what is equivalent

$$
\left(\frac{1+\zeta}{\zeta}-\frac{(1+\zeta)^{2} \theta^{2}}{\zeta}\right) T(E, \phi)^{2}+(1+2(1+\zeta) \theta) T(E, \phi)-\zeta \geq 0
$$

Thus, since by assumption, $T(E, \phi)>0$, either $\theta \geq \frac{1}{\sqrt{1+\zeta}}$ or $\theta<\frac{1}{\sqrt{1+\zeta}}$ and

$$
\begin{aligned}
2 \theta & \geq T(E, \phi) \geq \frac{-(1+2 \theta(1+\zeta))+\sqrt{1+4(1+\theta)(1+\zeta)}}{\frac{2(1+\zeta)}{\zeta}\left(1-(1+\zeta) \theta^{2}\right)} \\
\quad= & \frac{2 \zeta}{1+2 \theta(1+\zeta)+\sqrt{1+4(1+\theta)(1+\zeta)}} \geq \frac{2 \zeta}{1+2 \sqrt{1+\zeta}+\sqrt{5+4(\zeta+\sqrt{1+\zeta})}}
\end{aligned}
$$

on the interval $\left(0, \frac{1}{\sqrt{1+\zeta}}\right]$.

Step 2: $E_{0}$ is achieved.

Let $\left\{\left(E_{n}, \phi_{n}\right)\right\}$ be a minimizing sequence for $E_{0}$. Since $I_{V}=1+\zeta>1$, by (4.23), $\zeta\left\||| \phi_{n} \mid\right\|_{E_{n}}^{2} \leq(1+\zeta) T\left(E_{n}, \phi_{n}\right)$ for all $n$. Then, by the same arguments as in the proof of Lemma 4.5, we have

$$
\begin{aligned}
(1+\zeta) \int_{\mathbb{R}^{3}} V^{2}\left|\phi_{n}\right|^{2} d x & \leq\left.||\left|\phi_{n}\right|\right|_{E_{n}} ^{2} \leq \frac{(1+\zeta) T\left(E_{n}, \phi_{n}\right)}{\zeta} \\
& \leq \frac{2(1+\zeta)\left(E_{n}+1\right)}{\zeta}<\frac{2(1+\zeta)}{\zeta}
\end{aligned}
$$

This implies that

$$
\int_{\mathbb{R}^{3}} V^{2}\left|\phi_{n}\right|^{2} d x+\left.||\left|\phi_{n}\right|\right|_{E_{n}} \leq \frac{2(2+\zeta)}{\zeta}
$$

Therefore, up to subsequences, we can find $\phi_{0} \in L^{2}\left(\mathbb{R}^{3}\right)$ such that $\phi_{n} \underset{n \rightarrow+\infty}{\rightarrow} \phi_{0}$ weakly in $L^{2}\left(\mathbb{R}^{3}\right)$. Moreover, for all $\varepsilon>0$, there exists $R(\varepsilon)>0$ such that $\lim _{\varepsilon \rightarrow 0} R(\varepsilon)=+\infty$ and $\left\|\phi_{0}\right\|_{L^{2}(|x|>R(\varepsilon))} \leq \frac{\varepsilon}{2}$.

From (4.33), $\int_{\mathbb{R}^{3}} g_{E_{n}}^{-1}\left|L \varphi_{n}\right|^{2} d x \leq C$ for some constant $C>0$ independent of $n$. If $V$ is in $L^{\infty}\left(\mathbb{R}^{3}\right)$, this implies that $\left\{\varphi_{n}\right\}$ is bounded in $H^{1}\left(\mathbb{R}^{3}\right)$ and relatively compact in $L_{l o c}^{q}\left(\mathbb{R}^{3}\right)$ if $2 \leq q<6$. If on the contrary, $V(x)$ tends to $-\infty$ when $x$ approaches 0 , then $\left\{\varphi_{n}\right\}$ is only bounded in $H_{l o c}^{1}\left(\mathbb{R}^{3} \backslash\{0\}\right)$ and relatively compact in $L_{l o c}^{q}\left(\mathbb{R}^{3} \backslash\{0\}\right), 2 \leq q<6$. In all cases, $\left\{\phi_{n}\right\}$ is relatively bounded in $L_{l o c}^{q}\left(\mathbb{R}^{3} \backslash\{0\}\right), 2 \leq q<6$. Finally, one can use (4.33) to prove that $\left\{\phi_{n}\right\}$ is relatively compact in $L_{l o c}^{2}\left(\mathbb{R}^{3}\right)$ and that 


$$
\int_{\mathbb{R}^{3}} V\left|\phi_{n}\right|^{2} d x \underset{n \rightarrow \infty}{\longrightarrow} \int_{\mathbb{R}^{3}} V\left|\phi_{0}\right|^{2} d x
$$

Up to subsequences, we may consider now that $\lim _{n}\left\|\phi_{n}\right\|^{2}$ exists and we define

$$
M=\lim _{n}\left\|\phi_{n}\right\|^{2}-\left\|\phi_{0}\right\|^{2} .
$$

Let us now prove that

$$
\phi_{n} \underset{n \rightarrow \infty}{\longrightarrow} \phi_{0} \text { in } L^{2}\left(\mathbb{R}^{3}\right) .
$$

If $M=0,(4.36)$ holds. Assume, by contradiction, that $M>0$. By definition of $J^{+}$we have

$$
\left(\phi_{n}, \phi_{n}\right) J_{n}^{2}+2\left(\phi_{n},(1-V) \phi_{n}\right) J_{n}-\left(\phi_{n},(2-V) V \phi_{n}\right)-\left(\phi_{n}, H_{E_{0}} \phi_{n}\right)=0
$$

where by $J_{n}$ we denote $J^{+}\left(E_{n}, \phi_{n}\right)$. Now we consider a function $\chi_{\varepsilon} \in$ $C^{2}\left(\mathbb{R}^{3}\right)$ such that $\chi_{\varepsilon} \equiv 1$ on $B\left(0, \varepsilon^{-1}\right)$, supp $\chi_{\varepsilon} \subset B\left(0,2 \varepsilon^{-1}\right)$ and $\left\|\nabla \chi_{\varepsilon}\right\|_{L^{\infty}} \leq 2 \varepsilon$ and define $\phi_{n}^{\varepsilon}=\phi_{n} \chi_{\varepsilon}$. Obviously, the weak limit of $\phi_{n}^{\varepsilon}$ in $L^{2}\left(\mathbb{R}^{3}\right)$ is $\phi_{0}^{\varepsilon}:=\phi_{0} \chi_{\varepsilon}$. For $\varepsilon$ small enough, we obtain:

$$
\left(\phi_{n}, \phi_{n}\right)\left(J_{n}^{2}+2 J_{n}\right)-2\left(J_{n}+1\right)\left(\phi_{n}^{\varepsilon}, V \phi_{n}^{\varepsilon}\right)+\left(\phi_{n}^{\varepsilon}, V^{2} \phi_{n}^{\varepsilon}\right)-\left(\phi_{n}^{\varepsilon}, H_{E_{0}} \phi_{n}^{\varepsilon}\right)>C \varepsilon
$$

where $C$ is a negative constant independent of $n$ and $\varepsilon$. Then, we use the lower semicontinuity in $X$ of the quadratic form

$$
\phi \mapsto\left(\phi, H_{E_{0}} \phi\right)+(\phi, \phi)-\left(\phi, V^{2} \phi\right)
$$

and (4.34)-(4.35) to obtain, as $n \rightarrow+\infty$ and then as $\varepsilon \rightarrow 0$ :

$$
\begin{aligned}
\left(\left(\phi_{0}, \phi_{0}\right)+M\right)\left(\bar{J}^{2}+2 \bar{J}\right)-2(\bar{J}+1)\left(\phi_{0}, V \phi_{0}\right) & \\
& +\left(\phi_{0}, V^{2} \phi_{0}\right)-\left(\phi_{0}, H_{E_{0}} \phi_{0}\right) \geq 0,
\end{aligned}
$$

where $\bar{J}=\limsup _{n} J_{n}$.

Now, since $-1<\bar{J}<0$, if $M>0$ one has:

$$
M\left(\bar{J}^{2}+2 \bar{J}\right)<0 .
$$

Hence,

$(4.37)\left(\phi_{0}, \phi_{0}\right) \bar{J}^{2}+2\left(\phi_{0},(1-V) \phi_{0}\right) \bar{J}-\left(\phi_{0},(2-V) V \phi_{0}\right)-\left(\phi_{0}, H_{E_{0}} \phi_{0}\right)>\alpha>0$ 
which implies that for large $n$, either $J_{n}<J^{-}\left(E_{0}, \phi_{0}\right) \leq-1$, which is impossible, or $\underline{\lim }_{n} J_{n}>J^{+}\left(E_{0}, \phi_{0}\right)$, which contradicts the definition of $E_{0}:=\lim _{n} E_{n} \geq \varlimsup_{n} J_{n}$. Therefore, necessarily $M=0$ and $\phi_{n} \underset{n \rightarrow \infty}{\longrightarrow}$ $\phi_{0}$ in $L^{2}\left(\mathbb{R}^{3}\right)$. This, together with our assumptions on $V$ and lower semicontinuity implies that

$$
\begin{gathered}
\left(\phi_{0}, H_{E_{0}} \phi_{0}\right)+\left(\phi_{0}, \phi_{0}\right)-\left(\phi_{0}, V^{2} \phi_{0}\right) \leq \\
\leq \underline{\lim }_{n}\left(\left(\phi_{n}, H_{E_{n}} \phi_{n}\right)+\left(\phi_{n}, \phi_{n}\right)-\left(\phi_{n}, V^{2} \phi_{n}\right)\right), \\
\Delta\left(E_{0}, \phi_{0}\right) \leq \underline{\lim } \Delta\left(E_{n}, \phi_{n}\right) \leq 1,
\end{gathered}
$$

and therefore,

$$
J^{+}\left(E_{0}, \phi_{0}\right) \leq E_{0}
$$

and $\left(E_{0}, \phi_{0}\right)$ is a minimizer for $E_{0}$.

Step 3 : Constraint saturation.

Under our assumptions, it is straightforward to see that if $\left(E_{0}, \phi_{0}\right)$ is a minimum of problem (4.16), then $J^{+}\left(E_{0}, \phi_{0}\right)=E_{0}$, by definition of $E_{0}$ and because the map $E \mapsto J^{+}\left(E, \phi_{0}\right)$ is continuous. This also shows that

$$
E_{0}=\inf \left\{J^{+}(E, \phi) ; \phi \not \equiv 0,(E, \phi) \in(-1,0) \times X, J^{+}(E, \phi)=E\right\} .
$$

$\underline{\text { Step } 4}$ : Euler-Lagrange equations.

By classical computations, there is a Lagrange multiplier $\mu \in \mathbb{R}$ such that $\left(E_{0}, \phi_{0}\right)$ is a critical point of the functional $(1-\mu) E+\mu J^{+}$, i. e.,

$$
(1-\mu)+\mu \partial_{E} J^{+}\left(E_{0}, \phi_{0}\right)=0=\mu \partial_{\phi} J^{+}\left(E_{0}, \phi_{0}\right) .
$$

These two equalities show that $\partial_{\phi} J^{+}\left(E_{0}, \phi_{0}\right)=0$, or equivalently

$$
H_{E_{0}} \phi_{0}=\left(E_{0}-V\right)\left(E_{0}+2-V\right) \phi_{0} \quad \text { in } \mathcal{D}^{\prime}\left(\mathbb{R}^{3}\right)
$$

i.e., $\left(E_{0}, \phi_{0}\right)$ is a solution of (4.4).

It seems difficult to find a characterization of all potentials $V$ such that the assumptions of Theorem 4.3 hold. But, in order to fix our ideas about the validity range of this result, let us look at Coulomb potentials $-a|x|^{-1}$, $a>0$.

Proposition 4.6 . Let us consider $W_{1}(x):=-|x|^{-1}$. Then, for all $a \in$ $(0,1), D_{a W_{1}} \neq \emptyset$ and there is a constant $\zeta_{a}>0$ such that

$$
I_{a W_{1}}=1+\zeta_{a}>1,
$$


and therefore, for all $a<1$ the smallest eigenvalue of $H-\frac{a}{|x|}$ in the interval $(-1,1)$ is given by the infimum of $E$ over the set $D_{a W_{1}}$.

\section{Proof of Proposition 4.6.}

It is well known that for all $a<1$ there exists a smallest eigenvalue of $H-\frac{a}{|x|}$ in the gap of the essential spectrum, $\lambda_{1}^{a}>0$. Moreover, when $a$ approaches $1, \lambda_{1}^{a}$ converges to 0 . This implies the existence of a minimal $E_{a}=\lambda_{1}^{a}-1$ in $(-1,0)$ (given by the minimization method of Theorem 4.3) and $\phi_{a} \in X \backslash\{0\}$ such that $\left(E_{a}, \phi_{a}\right)$ is a solution of equation (4.4).

The map $a \mapsto I_{a W_{1}}$ is continuous. Assume, by contradiction, that $I_{A_{1} W_{1}}=1$ for some $A_{1}<1$. Then, there exist $E_{n} \in(-1,0)$, $a_{n} \rightarrow A_{1}$ and $\varphi_{n}$ in $X_{0}$ such that $J^{+}\left(E_{n}, \phi_{n}\right) \leq E_{n}$ and

$$
\int_{\mathbb{R}^{3}} \frac{\left|L \varphi_{n}\right|^{2}+\left|\varphi_{n}\right|^{2}}{E_{n}+2+a_{n}\left|W_{1}\right|}=\left(1+\frac{1}{n}\right) \int_{\mathbb{R}^{3}} \frac{a_{n}^{2} W_{1}^{2}\left|\varphi_{n}\right|^{2}}{E_{n}+2+a_{n}\left|W_{1}\right|}
$$

By homogeneity, we can choose the sequence $\left\{\varphi_{n}\right\}$ to satisfy

$$
\int_{\mathbb{R}^{3}} \frac{W_{1}^{2}\left|\varphi_{n}\right|^{2}}{E_{n}+2+a_{n}\left|W_{1}\right|}=1 \text { for all } n .
$$

Then, by the definition of $J^{+}$we have

$$
\begin{aligned}
-1<J^{+}\left(E_{n}, \phi_{n}\right) & =-1+\frac{a_{n}\left(\phi_{n}, W_{1} \phi_{n}\right)+\sqrt{a_{n}^{2}\left(\phi_{n}, W_{1} \phi_{n}\right)^{2}+\frac{a_{n}^{2}}{n}\left\|\phi_{n}\right\|^{2}}}{\left\|\phi_{n}\right\|^{2}} \\
& \leq-1+\frac{a_{n}}{\sqrt{n} \sqrt{\left(\phi_{n}, \phi_{n}\right)}} .
\end{aligned}
$$

Now, if $\underline{\lim }_{n}\left(\phi_{n}, \phi_{n}\right)>0,(4.44)$ implies that $\lim _{n} J^{+}\left(E_{n}, \phi_{n}\right)=-1$, and then for $n$ large, the minimization of $J^{+}$on $D_{a_{n} W_{1}}$ provides us with an eigenvalue of $H-a_{n} W_{1}$ smaller than the minimum eigenvalue of this operator, $\lambda_{1}^{a}$. So, up to subsequences one must have

$$
\lim _{n}\left(\phi_{n}, \phi_{n}\right)=0
$$

Let us show that this is also impossible and so we will have proved that $A_{1}=1$.

If (4.45) holds, we can take a cut-off function $\chi_{r} \in C^{1}\left(\mathbb{R}^{3}\right), \chi_{r} \equiv 1$ on $B(0, r)$, supp $\chi_{r} \subset B(0,2 r)$ such that for $r$ small and $n$ large, we have

$$
\int_{\mathbb{R}^{3}} \frac{\left|L\left(\varphi_{n} \chi_{r}\right)\right|^{2}}{\left|W_{1}\right|} d x \leq \beta A_{1} \int_{\mathbb{R}^{3}}\left|W_{1}\right|\left|\varphi_{n} \chi_{r}\right|^{2} d x
$$


for some $\beta \in\left(1, A_{1}^{-1}\right)$, which is impossible, because for the Coulomb potential one has

$$
\inf _{\varphi \in H^{1 / 2}\left(\mathbb{R}^{3}\right)}\left(\int_{\mathbb{R}^{3}}|x||L \varphi|^{2}\right)\left(\int_{\mathbb{R}^{3}}|x|^{-1}|\varphi|^{2}\right)^{-1}=1
$$

and $A_{1}$ was supposed to be strictly less than 1 .

\section{References}

[1] W.E. Bayliss, S. J. Peel. Stable variational calculations with the Dirac Hamiltonian. Physical Review A, 28 (4) (1983), p. 2552-2554.

[2] C. Bennet, R. Sharpley. Interpolation of Operators. Academic Press, 1988 .

[3] A. Berthier, V. Georgescu. Sur la propriété de prolongement unique pour l'opérateur de Dirac. C. R. Acad. Sci. Paris, Sér. A 291 (1980), p. 603-606.

[4] A. Berthier, V. Georgescu. Sur le spectre ponctuel de l'opérateur de Dirac. C. R. Acad. Sci. Paris, Sér. A 297 (1983), p. 335-338.

[5] A. Berthier, V. Georgescu. On the point spectrum for Dirac operators. J. Func. Anal. 71 (1987), p. 309-338.

[6] J.D. Bjorken-S.D. Drell. Relativistic quantum fields. McGraw-Hill (1965).

[7] G.E. Brown, D.G. Ravenhall. On the interaction of two electrons. Proc. Roy. Soc. London A 208 (A 1095) (1951), p. 552-559.

[8] V.I. Burenkov, W.D. Evans. On the evaluation of the norm of an integral operator associated with the stability of one-electron atoms. Math. Phys. Preprint archive mp-arc (97-247) (1997).

[9] S.N. Datta, G. Deviah. The minimax technique in relativistic HartreeFock calculations. Pramana 30(5) (1988), p. 393-416.

[10] I. Daubechies, E.H. Lieb. One-electron relativistic molecules with Coulomb interaction. Comm. Math. Phys. 90(4) (1983), p. 497-510. 
[11] I. Daubechies, E.H. Lieb. Relativistic molecules with Coulomb interaction, in Differential equations, I. Knowles and R. Lewis ed., p. 143-148, North-Holland Math. Stud., 92, North-Holland, Amsterdam-New York, 1984 .

[12] G.W.F. Drake, S.P. Goldman. Relativistic sturmian and finite basis set methods in atomic physics. Adv. Atomic Molecular Phys. 23(1988), p. 23-29.

[13] Ph. Durand. Transformation du Hamiltonien de Dirac en Hamiltoniens variationnels de type Pauli. Application à des atomes hydrogenoïdes. C. R. Acad. Sc. Paris 303 , série II, numéro 2 (1986), p. 119-124.

[14] Ph. Durand, J.-P. Malrieu. Effective Hamiltonians and pseudo potentials as tools for rigorous modelling. In Ab initio methods in Quantum Chemistry I. K.P. Lawley ed.. J. Wiley and sons, 1987.

[15] M.J. Esteban, E. Séré. Existence and multiplicity of solutions for linear and nonlinear Dirac problems. Partial Differential Equations and Their Applications. CRM Proceedings and Lecture Notes, volume 12. Eds. P.C. Greiner, V. Ivrii, L.A. Seco and C. Sulem. AMS, 1997.

[16] W.D. Evans, P. Perry, H. Siedentop. The spectrum of realtivistic oneelectrom atoms according to Bethe and Salpeter. Comm. Math. Phys. 178(3) (1996), p. 733-746.

[17] M. Griesemer, H. Siedentop. A minimax principle for the eigenvalues in spectral gaps. To appear in J. London Math. Soc.

[18] G. Hardekopf, J. Sucher. Critical coupling constants for relativistic wave equations and vacuum breakdown in quantum electrodynamics. Phys. Rev. A 31(4) (1985), p. 2020-2029.

[19] I.W. Herbst. Spectral theory of the operator $\left(p^{2}+m^{2}\right)^{1 / 2}-Z e^{2} / r$. Comm. Math. Phys. 53 (1977), p. 285-294.

[20] R.N. Hill, C. Krauthauser. A solution to the problem of variational collapse for the one-particle Dirac equation. Phys. Rev. Lett. 72(14) (1994), p. 2151-2154.

[21] T. Kato. Perturbation theory for linear operators. Springer, 1966.

[22] W. Kutzelnigg. Relativistic one-electron Hamiltonians for electrons only' and the variational treatment of the Dirac equation. Chem. Phys. (1997). 
[23] A. Le Yaouanc, L. Oliver, J.-C. Raynal. The Hamiltonian $\left(p^{2}+m^{2}\right)^{1 / 2}-$ $\alpha / r$ near the critical value $\alpha_{c}=2 / \pi$. J. Math. Phys. 38(8) (1997), p. 3397-4012.

[24] E. van Lenthe, R. van Leeuwen, E.J. Baerends, J.G. Snijders. Relativistic regular two-component Hamiltonians. In New challenges in computational Quantum Chemistry. R. Broek et al ed.. Publications Dept. Chem. Phys. and Material sciences. University of Groningen, 1994.

[25] Selecta of E. H. Lieb. The stability of matter: from atoms to stars. Edited by W. Thirring (second edition), Springer.

[26] E.H. Lieb, H. Siedentop, J.P. Solovej. Stability and instability of relativistic electrons in classical electromagnetic fields. J. Stat. Phys. (1997).

[27] E.H. Lieb, H. Siedentop, J.P. Solovej. Stability of relativistic matter with magnetic fields. Phys. Rev. Lett. 79(10) (1997), p. 1755-1758.

[28] A. Messiah. Mécanique quantique II. Masson, Paris, 1960.

[29] M. Reed, B. Simon. Methods of Modern Mathematical Physics. Academic Press, New York, 1978.

[30] J.D. Talman. Minimax principle for the Dirac equation. Phys. Rev. Lett. 57(9) (1986), p. 1091-1094.

[31] B. Thaller. The Dirac equation. Springer-Verlag, 1992.

[32] C. Tix. Strict positivity of a relativistic Hamiltonian due to Brown and Ravenhall. Bull. London Math. Soc. (1998).

[33] C. Tix. Lower bound for the ground state energy of the no-pair Hamiltonian. Physics Letters B 405 (1997), p. 293-296. 